\title{
In vitro and in vivo enzymatic dephosphorylation of phytate in maize-soya bean meal diets for broiler chickens by phytase of Mitsuokella jalaludinii.
}

\begin{abstract}
The efficacy of Mitsuokella jalaludinii phytase to dephosphorylate phytate in maize-soya bean meal diets was evaluated by two in vitro simulation procedures and an in vivo balance study in which the difference between the total phosphorus $(\mathrm{P})$ intake and total $\mathrm{P}$ in the total excreta collected was determined. Using the first in vitro method, which was a three-step procedure simulating the physiological parameters of the intestinal tract (crop, stomach and intestine), a quadratic ( $\mathrm{P}<0.001)$ increase of released $\mathrm{P}$ was observed in diets containing $2.4 \mathrm{~g}$ available $\mathrm{P}(\mathrm{aP}) / \mathrm{kg}$ and $4.6 \mathrm{~g} \mathrm{aP} / \mathrm{kg}$ when $250-2500 \mathrm{U}$ M. jalaludinii phytase $/ \mathrm{kg}$ were added to the feed. Using the second in vitro method, which consisted of the three-step simulation procedure described above plus an additional dialysis step under conditions simulating gradient absorption in the small intestine, $\mathrm{P}$ was also released from the feed quadratically $(\mathrm{P}<0.001)$ when $250-2500 \mathrm{U}$ M. jalaludinii phytase $/ \mathrm{kg}$ were added to the diet containing either level of aP. The results from the in vivo study showed that M. jalaludinii phytase supplementation increased the coefficient of total tract apparent digestibility (CTTAD) of $P$ $(\mathrm{P}<0.001)$, crude protein $(\mathrm{CP} ; \mathrm{P}=0.004)$ and dry matter $(\mathrm{DM} ; \mathrm{P}<0.001)$ in the diet of 42 -d-old broiler chickens. After the Hanes transformation [i.e. the in vivo response measurement divided by the in vitro released $\mathrm{P}$ was treated as the dependent variable ( $\mathrm{Y}$ ), and in vitro released $\mathrm{P}$ was treated as the independent variable $(\mathrm{X})$, in the regression analysis], the $\mathrm{P}$ released following simulation of digestion in the crop using the first in vitro method or the $\mathrm{P}$ released using the second in vitro method, correlated to in vivo CTTAD of $\mathrm{P}(\mathrm{R} 2=0.70$, $\mathrm{P}=0.0027$; or $0.71 \mathrm{P}=0.0024$, respectively), $\mathrm{CP}(\mathrm{R} 2=0.89, \mathrm{P}<0.0001$; or $0.88, \mathrm{P}<0.0001$, respectively), $\mathrm{DM}(\mathrm{R} 2=0.93, \mathrm{P}<0.0001$; or $0.89, \mathrm{P}<0.0001$, respectively $)$ and apparent metabolizable energy (AME; R2 $=0.92, \mathrm{P}<0.0001$; or $0.88, \mathrm{P}<0.0001$, respectively). About $75.0-95.8 \%$ of the supplemental M. jalaludinii phytase activity was found in the contents of the crop of broiler chickens and only $5.6-18 \%$ of the activity was found in the stomach. It is concluded that $M$. jalaludinii phytase supplementation significantly increased the dephosphorylation of phytate both in vitro and in vivo. The responses in vivo of $\mathrm{P}, \mathrm{CP}, \mathrm{DM}$ and AME digestibility to $\mathrm{M}$. jalaludinii phytase supplementation could be predicted by the $\mathrm{P}$ released in vitro by $\mathrm{M}$. jalaludinii phytase supplementation. The M. jalaludinii phytase was mostly active in the crop of broiler chickens and its activity was not affected in vivo by the aP level of the feed.
\end{abstract}

Keyword: Mitsuokella jalaludinii; Phytase; Phytate dephosphorylation; Broiler. 